\title{
Die sich wandelnde Rolle von Daten in Organisationen: Von der elektronischen Datenverarbeitung zum „Daten-Business“
}

\author{
Susanne Strahringer • Martin Wiener
}

Angenommen: 14. April 2021

(C) Der/die Autor(en) 2021

Every company has big data in its future, and every company will eventually be in the data business (Thomas H. Davenport).

In der Praxis und auch in der Wissenschaft spielen Daten zunehmend eine zentrale und erfolgskritische Rolle. Wenngleich diese Entwicklung in Verbindung mit der rasanten Verbreitung von Schlagwörtern wie „Big Data“ und datengetriebener Wertschöpfung teils als ein disruptiver Trend wahrgenommen wird, stellt sie im Grunde eine konsequente Fortführung bisheriger Entwicklungen dar, die möglicherweise - wie von Thomas H. Davenport angedeutet - darin münden wird, dass das Gros der Unternehmen im „Daten-Business“ tätig sein wird. Bezeichnend für diesen nächsten logischen Entwicklungsschritt ist die steigende Anzahl von datengetriebenen Geschäftsmodellen, in denen Daten die Rolle einer strategischen Kernressource einnehmen.

Beim Nachzeichnen der bisherigen Entwicklungsschritte fällt auf, dass die Bedeutung von Daten in der Ära der elektronischen Datenverarbeitung - also zu einer Zeit als man noch nicht von IT, sondern von der EDV sprach - im Vergleich zu heute als eher gering einzustufen ist. Auch wenn Daten bereits in den Anfängen der betriebswirtschaftlichen EDV eine wichtige Rolle spielten, war man damals schon zufrieden, wenn interne Daten erfasst und in „Batches“ mithilfe von einfachen EVA-Routinen (Eingabe-Verarbeitung-Ausgabe) verarbeitet werden konnten. Zu dieser Zeit wurden Daten folglich in erster Linie als Input und Output von Geschäftstransaktionen

\footnotetext{
S. Strahringer $(\bowtie)$

Technische Universität Dresden, Dresden, Deutschland

E-Mail: susanne.strahringer@tu-dresden.de

M. Wiener

Technische Universität Dresden, Dresden, Deutschland

E-Mail: martin.wiener@tu-dresden.de
} 
verstanden; die Ableitung von höherwertigen Erkenntnissen bzw. die Schaffung von neuen Werten im Allgemeinen stand hingegen noch nicht im Fokus.

Mit der Einsicht, dass integrierte Daten - also Datenbestände, die konsistent aufeinander Bezug nehmen - nützlicher sind als isolierte Datenbestände, kam es zu einer Reihe von weiteren Entwicklungen. Zur Integration von Daten sind zunächst funktional gegliederte Anwendungssysteme entstanden. Die Datenintegration wurde dann alsbald als wesentlicher „Enabler“ der Prozessintegration erkannt, so dass sich funktional gegliederte Anwendungssysteme zunehmend hin zu prozessorientierten Systemen entwickelt haben, so wie dies bei zeitgemäßen Enterprise Resource Planning (ERP)-Systemen üblich ist. Die hohe Bedeutung von Daten für die Unterstützung von Geschäftsprozessen ist bis heute ungebrochen, zumal Daten nicht mehr nur als Prozessinput und -output angesehen werden, sondern auch als Informationsquelle über Prozesse, die zur gezielten und systematischen Verbesserung eben dieser genutzt werden kann.

In einem weiteren Entwicklungsschritt haben Daten eine zunehmende Bedeutung für die Geschäftsproduktunterstützung erlangt. In diesem Zusammenhang sind unter anderem datenbasierte Konzepte der „Mass Customization“ und Ansätze zur Individualisierung oder Personalisierung von Kundenangeboten sowie der Trend hin zur sog. „Servitization“ (also zum Angebot von physischen Produkten in Form von Services) mitsamt der daraus resultierenden Entstehung von komplexen ProduktService-Systemen zu nennen. Insofern ist es wenig überraschend, dass viele Unternehmen derzeit versuchen, ihre traditionellen Geschäftsmodelle zu transformieren und Daten verstärkt zur Unterstützung ihrer Produkte und zugleich zur Verbesserung ihrer Prozesse zu nutzen. Diese Unternehmen bzw. die ihnen zugrundliegenden Geschäftsmodelle werden im vorliegenden Schwerpunktheft als Datennutzer bezeichnet.

Neben der Datennutzung zur Geschäftsprozess- und Geschäftsproduktunterstützung werden Daten aber auch immer öfter als das eigentliche Geschäftsprodukt angesehen. Stehen hierbei die Daten selbst im Mittelpunkt des Wertangebots eines Geschäftsmodells, so sprechen wir von Datenlieferanten (Daten als immaterielle Ware); stehen Services an Daten im Vordergrund, sprechen wir von Datenbefähigern (Daten als Objektfaktor eines Services).

Betrachtet man die oben genannten Geschäftsmodelltypen aus einer Prozesssicht, werden auch die engen Bezüge zwischen diesen drei Typen deutlich. Stark vereinfacht kann dies so aussehen: Datennutzer, die selbst nicht über die benötigten Datenressourcen und/oder -fähigkeiten verfügen, können zum einen über Datenlieferanten relevante Daten beziehen und zum anderen die Expertise und Software-/ Infrastrukturlösungen von Datenbefähigern nutzen, um letztendlich Wert aus Daten zu generieren. An dieser Stelle ist anzumerken, dass Datenbefähiger natürlich auch Datenlieferanten oder andere Datenbefähiger unterstützen können.

Aufbauend auf bestehenden Forschungsarbeiten gehen wir im ersten Beitrag dieses Schwerpunktheftes zunächst ausführlicher auf die oben genannte Typologie von datengetriebenen Geschäftsmodellen ein (siehe Grundlagenbeitrag von Strahringer und Wiener) und leiten ein konzeptuelles Rahmenwerk ab, das zur Analyse und Beschreibung entsprechender Geschäftsmodelle herangezogen werden kann. Dass eine Typologie eher auf Rein- oder Idealformen abstellt, die in der Realität in die- 
ser Eindeutigkeit oftmals nicht vorkommen, zeigt sich zum Teil auch in den sich anschließenden Beiträgen dieses Heftes, in denen Mischformen der drei Geschäftsmodelltypen aufgegriffen werden oder in denen das Augenmerk auf dem Zusammenspiel verschiedener Typen liegt.

Die Reihenfolge der einzelnen Heftbeiträge orientiert sich an einer „Data Supply Chain“-Perspektive auf datengetriebene Geschäftsmodelle: Beginnend mit der Untersuchung von Datenlieferanten verlagert sich der inhaltliche Fokus der Heftbeiträge schrittweise in Richtung von Datennutzern und von dort hin zu Datenbefähigern und schließlich zurück zu Datenlieferanten-Geschäftsmodellen. So stellen Kramberg und Heinzl das Konzept der Datenplattformökosysteme vor und verdeutlichen dieses anhand von Praxisbeispielen. Im Beitrag von Wulf und Betz wird dann das Zusammenspiel von Krankenversicherungen (als Datennutzer) mit Datenökosystemen in der Form von Gesundheitsplattformen (als Datenlieferanten) untersucht und kritisch beleuchtet, wohingegen im Beitrag von Rüdian und Vladova am Beispiel einer Plattform für Online-Kurse dargestellt wird, wie Datennutzer- und Datenlieferanten-Geschäftsmodelle synergetisch kombiniert werden können und warum dies im vorliegenden Fall für die Nachhaltigkeit der Kursplattform auch notwendig ist. Der nachfolgende Beitrag von Vogt et al. fokussiert explizit auf Datennutzer und zeigt auf, dass datengetriebene Geschäftsmodelle auch von kleinen und mittleren Unternehmen in traditionellen Branchen, wie z. B. im Maschinen- und Anlagenbau, erfolgsversprechend verfolgt werden können. Darüber hinaus wird im Beitrag von Kortum et al. beschrieben, wie aus dem Datennutzer-Geschäftsmodell einer Autowerkstatt durch Verallgemeinerung und dem Angebot von ,as-a-Service“-Lösungen ein Datenbefähiger-Geschäftsmodell entwickelt werden kann. Der Beitrag von Nissen schlägt dann die Brücke hin zu reinen Datenbefähiger-Geschäftsmodellen im Consulting-Kontext. Den Kreis schließen zwei Beiträge, die an der Schnittstelle zwischen Datenbefähigern und Datenlieferanten angesiedelt sind: Arlinghaus et al. beleuchten den Status quo von Geschäftsmodellen für öffentliche und privatwirtschaftliche Datentreuhandstellen; Heinbach et al. beschreiben ein Geschäftsmodell für das datengetriebene Transportmanagement. Vervollständigt wird der thematische Schwerpunkt des Heftes mit zwei methodischen Beiträgen: Während Förster et al. ein Vorgehensmodell zur Wertrealisierung in datengetriebenen Geschäftsmodellen vorstellen, liegt der Fokus bei Häckel et al. auf der Entwicklung von datengetriebenen Services.

Wie bei HMD-Heften üblich wird auch dieses Schwerpunktheft abgerundet mit einer Auswahl von Spektrumsbeiträgen und zwei Buchbesprechungen.

An dieser Stelle möchten wir uns recht herzlich bei allen Autor:innen dieser Ausgabe bedanken, die ihr Wissen mit uns und Ihnen teilen und auf diesem Wege wertvolle Einblicke in die Entwicklung und Gestaltung datengetriebener Geschäftsmodelle geben - und zugleich durch kritische Hinweise und Diskussion wichtige Denkanstöße liefern.

Wir wünschen allen Leser:innen eine spannende Lektüre und hoffen, dass Sie neue Erkenntnisse aus den Beiträgen ziehen und einige Impulse für sich aufgreifen und ,verwerten“ können. Auch wenn dabei nicht immer ein neues Geschäftsmodell entstehen wird, so doch vielleicht die eine oder andere neue Idee und damit mögli- 
cherweise ein erster Schritt in Richtung Ihres eigenen „Daten-Business“. Wenn dem so sein sollte, viel Erfolg dabei!

\section{Susanne Strahringer \\ Martin Wiener}

Funding Open Access funding enabled and organized by Projekt DEAL.

Open Access Dieser Artikel wird unter der Creative Commons Namensnennung 4.0 International Lizenz veröffentlicht, welche die Nutzung, Vervielfältigung, Bearbeitung, Verbreitung und Wiedergabe in jeglichem Medium und Format erlaubt, sofern Sie den/die ursprünglichen Autor(en) und die Quelle ordnungsgemäß nennen, einen Link zur Creative Commons Lizenz beifügen und angeben, ob Änderungen vorgenommen wurden.

Die in diesem Artikel enthaltenen Bilder und sonstiges Drittmaterial unterliegen ebenfalls der genannten Creative Commons Lizenz, sofern sich aus der Abbildungslegende nichts anderes ergibt. Sofern das betreffende Material nicht unter der genannten Creative Commons Lizenz steht und die betreffende Handlung nicht nach gesetzlichen Vorschriften erlaubt ist, ist für die oben aufgeführten Weiterverwendungen des Materials die Einwilligung des jeweiligen Rechteinhabers einzuholen.

Weitere Details zur Lizenz entnehmen Sie bitte der Lizenzinformation auf http://creativecommons.org/ licenses/by/4.0/deed.de. 